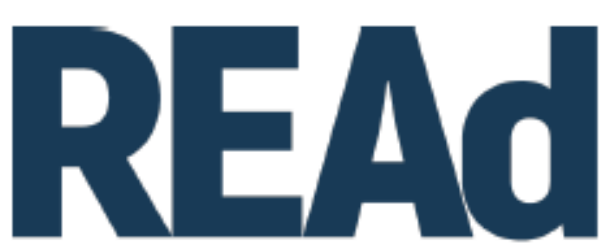

Revista Eletrônica de Administração

\title{
ORGANIZAÇÃO DO SERVIÇO DE TELECUIDADO FARMACÊUTICO COMO ESTRATÉGIA DE COMBATE À COVID-19 NO RIO GRANDE DO SUL ${ }^{1}$
}

\author{
Agnes Nogueira Gossenheimer ${ }^{2}$ \\ Ana Paula Rigo ${ }^{3}$ \\ Roberto Eduardo Schneiders ${ }^{4}$
}

http://dx.doi.org/10.1590/1413-2311.293.109474

\begin{abstract}
RESUMO
O Telecuidado Farmacêutico consiste na prestação de serviços farmacêuticos clínicos de forma remota, através da utilização de tecnologias de informação e telecomunicações, tendo o potencial de melhorar resultados em saúde e reduzir os problemas relacionados ao uso de medicamentos. As vantagens adicionais da telefarmácia incluem o custo-benefício, possibilitando o acesso ao serviço de acompanhamento farmacêutico e a possibilidade de atingir pessoas em localidades remotas e em condições de saúde que limitam a mobilidade. Considerando esse novo paradigma de oferta de serviços farmacêuticos, bem como o atual quadro pandêmico causado pelo novo coronavírus 2019 (COVID-19), em 5 de maio de 2020 foi lançado pela Secretaria de Estado da Saúde do Rio Grande do Sul, um programa de implantação do Cuidado Farmacêutico, CUIDAR +, e o primeiro serviço: Telecuidado Farmacêutico. O objetivo deste estudo é apresentar como o serviço de Telecuidado Farmacêutico foi estruturado e ofertado no combate à COVID-19 como uma estratégia emergente. O serviço foi desenvolvido a partir da avaliação das necessidades em saúde dos usuários da Farmácia de Medicamentos Especiais de Porto Alegre e definido por meio de modelagem de serviços. Foi organizado para acompanhar os pacientes com doenças

\footnotetext{
${ }^{1}$ Recebido em 9/11/2020, aceito em 30/11/2020.

2 Secretaria de Estado da Saúde do Rio Grande do Sul - Porto Alegre, RS (Brasil); agnesgossenheimer@saude.rs.gov.br; https://orcid.org/0000-0002-7424-8426.

${ }^{3}$ Secretaria de Estado da Saúde do Rio Grande do Sul - Porto Alegre, RS (Brasil); https://orcid.org/0000-00029142-9421; ana-rigo@ saude.rs.gov.br.

${ }^{4}$ Secretaria de Estado da Saúde do Rio Grande do Sul - Porto Alegre, RS (Brasil); https://orcid.org/0000-00030135-2844; roberto-schneiders@ saude.rs.gov.br.
} 
respiratórias crônicas, orientando em relação ao uso correto dos medicamentos, otimizando a adesão ao tratamento e orientando em relação às dúvidas relacionadas à COVID-19.

Palavras-chaves: Telessaúde. Cuidado Farmacêutico. COVID-19. Asma. Telecuidado.

\section{ORGANIZATION OF THE TELE-PHARMACEUTICAL CARE SERVICE AS AN EMERGING IN THE FIGHT AGAINST COVID-19 IN RIO GRANDE DO SUL}

Tele-Pharmaceutical Care consists of providing clinical pharmaceutical services remotely, through the use of information and telecommunications technologies, with the potential to improve health outcomes and reduce problems related to the use of medicines. The additional advantages of telepharmacy include cost-effectiveness, enabling access to the pharmaceutical monitoring service and the possibility of reaching people in remote locations and in health conditions that limit mobility. Considering this new paradigm for the provision of pharmaceutical services, as well as the current pandemic picture issued by the new coronavirus 2019 (COVID-19), on May 5, 2020 was launched by the State Health Departament of the State of Rio Grande do Sul, a program for the implementation of Pharmaceutical Care, CUIDAR +, and the first service: Pharmaceutical Care. The aim of this study is to present how the Pharmaceutical Tele-care service was structured and offered to combat COVID-19 as an emerging strategy. The service was developed based on the evaluation of the health needs of users of the Special Medicines Pharmacy in Porto Alegre and defined through service modeling. Patients with chronic respiratory diseases were organized to follow up, providing guidance regarding the correct use of medications, optimizing adherence to treatment and providing guidance regarding questions related to COVID-19.

Keywords: Telehealth. Pharmaceutical care. COVID-19. Asthma. Telecare.

\section{ORGANIZACIÓN DEL SERVICIO DE TELECUIDADO FARMACEUTICO COMO ESTRATEGIA EN LA LUCHA CONTRA COVID-19 EN EL RIO GRANDE DO SUL}

El telecuidado farmaceutico consiste en la prestación de servicios farmacéuticos clínicos de forma remota, mediante el uso de tecnologías de la información y las telecomunicaciones, con el potencial de mejorar los resultados de salud y reducir los problemas relacionados con el uso de medicamentos. Las ventajas adicionales del telemarketing incluyen la rentabilidad, que 
permite el acceso al servicio de monitorización farmacéutica y la posibilidad de llegar a personas en ubicaciones remotas y en condiciones de salud que limitan la movilidad. Este nuevo paradigma para la prestación de servicios farmacéuticos, así como el actual cuadro pandémico emitido por el nuevo coronavirus 2019 (COVID-19), el 5 de mayo de 2020 fue lanzado por la Secretaría de Estado del Estado de Rio Grande do Sul, un programa de implementación de Atención Farmacéutica, CUIDAR +, y el primer servicio: Atención Farmacéutica. El objetivo de este estudio es presentar cómo se estructuró y ofreció el servicio de Teleasistencia Farmacéutica para combatir el COVID-19 como uns estratégia emergente. El servicio se desarrolló a partir de la evaluación de las necesidades de salud de los usuarios de la Farmacia de Medicamentos Especiales de Porto Alegre y se definió mediante el modelado de servicios. Se organizó el seguimiento de los pacientes con enfermedades respiratorias crónicas, brindando orientación sobre el uso correcto de los medicamentos, optimizando la adherencia al tratamiento y brindando orientación sobre cuestiones relacionadas con el COVID-19.

Palabras clave: Telesalud. Servicios Farmacéuticos. COVID-19. Asma. Telecuidado.

\section{INTRODUÇÃOO}

No contexto da pandemia de COVID-19, houve um desenvolvimento acelerado na organização dos serviços de saúde por atendimento remoto, como é o caso, por exemplo, da implementação do Telecuidado Farmacêutico implementado pela Secretaria Estadual da Saúde do Rio Grande do Sul (SES/RS). Segundo Mintzberg (2010, p.27), uma estratégia de caráter emergente é aquela na qual “[...] um padrão realizado não era expressamente pretendido". Ainda que já houvesse um plano deliberado para implementar o Telecuidado Farmacêutico no Estado, a situação emergencial de saúde modificou a urgência da execução deste serviço, forçando os planejadores a adotarem novas perspectivas para a formulação estratégica.

O relato abaixo é baseado em fatos reais e ilustra o funcionamento do serviço:

"Em meio à pandemia, um idoso que recebeu uma prescrição para seus medicamentos para asma, pela primeira vez foi até a Farmácia do Estado receber seu tratamento. Lá chegando o farmacêutico dispensou os medicamentos prescritos e lhe passou algumas orientações, mas, em situação de distanciamento social, foi difícil verificar questões aprofundadas sobre sua condição de saúde e saber as possíveis dúvidas do paciente. Depois de uma semana, um 
farmacêutico certificado liga para a pessoa e revisa o uso dos medicamentos, retomando as informações fornecidas e verificando a técnica de administração. Nesse momento, o paciente demonstra que estava com dúvidas e utilizando de forma incorreta o medicamento para asma. O farmacêutico constrói um plano de cuidado junto com a pessoa e sugere intervenções educativas necessárias para o caso. Depois de 1 mês, esse paciente volta a ser contatado para que seja realizado o monitoramento da doença e reforçadas as orientações. Nesse período de interconsulta médica, esse paciente pode contar com o apoio do profissional farmacêutico no que diz respeito a seus medicamentos, melhorando assim a adesão ao tratamento e o controle da doença."

Esse cenário representa como a telefarmácia tem o potencial de colaborar para o Sistema Único de Saúde (SUS), aumentando a efetividade do tratamento ao indivíduo. Ainda assim, sua implementação depende da superação de barreiras e de mudanças na organização de como os serviços farmacêuticos são prestados.

Neste artigo, serão apresentadas definições sobre telessaúde, telefarmácia, telecuidado farmacêutico e estratégia emergente, seguido de um relato da organização inicial de um teleserviço farmacêutico na SES/RS, contando com o envolvimento direto dos autores deste trabalho. Para isso, a coleta de dados foi realizada mediante observação participante. Será demonstrado como a construção de uma organização como essa, em meio a um estado de calamidade pública, só é possível a partir de uma prática administrativa que reconhece a necessidade de formar a estratégia durante a implementação do serviço.

\section{TELESSAÚdE, TELEFARMÁCIA E TELECUIDADO FARMACÊUTICO}

Telessaúde é o uso de tecnologias de informação e telecomunicações para fornecer assistência e educação em saúde, conectando usuários e profissionais que estão em locais diferentes. Conforme a definição da OMS, telessaúde é a prestação de serviços de saúde, onde pacientes e prestadores estão separados por distância. Abrange uma definição ampla de serviços de saúde com tecnologia habilitada, incluindo telemedicina (diagnóstico e tratamento de doença ou lesão) e serviços como avaliação, monitoramento, comunicação, prevenção e educação. A telessaúde pode contribuir para alcançar a cobertura universal de saúde, melhorando o acesso dos pacientes a serviços de saúde de qualidade e de baixo custo, onde

quer que estejam. É particularmente valiosa para aqueles que residem em áreas remotas, grupos vulneráveis e populações em envelhecimento (WHO, 2005). Em 16 de abril de 2020 foi 
sancionada a Lei 13.989 (BRASIL, 2020), que estabelece a utilização da telemedicina durante a crise do novo coronavírus. A lei autoriza o uso da telemedicina durante a pandemia "em caráter emergencial" e define a modalidade como "o exercício da medicina mediado por tecnologias para fins de assistência, pesquisa, prevenção de doenças e lesões e promoção de saúde”. O texto também estabelece que o médico deverá informar ao paciente todas as limitações inerentes ao uso da telemedicina, "tendo em vista a impossibilidade de realização de exame físico durante a consulta", e que "a prestação de serviço de telemedicina seguirá os padrões normativos e éticos usuais do atendimento presencial, inclusive em relação à contraprestação financeira pelo serviço prestado, não cabendo ao poder público custear ou pagar por tais atividades quando não for exclusivamente serviço prestado ao Sistema Único de Saúde (SUS)". A questão que emerge disso é: a Telessaúde veio para ficar?

Dentro do contexto da telessaúde, se insere a telefarmácia, que consiste na prestação de serviços farmacêuticos de forma remota a um paciente, por meio do uso de telecomunicações e outras tecnologias. A telefarmácia oferece uma série de oportunidades para melhorar os cuidados de saúde e os resultados para pacientes (BLUM, 2016). Os usos atuais da telefarmácia incluem aconselhamento ao paciente por telefone, gerenciamento de terapia medicamentosa, gerenciamento colaborativo de medicamentos, orientação em relação ao acesso à medicamentos, supervisão remota de dispensação e sistemas automáticos de dispensação (TRAYNOR, 2010). Esse tipo de serviço já foi implementado na Austrália, Estados Unidos e Canadá, por exemplo, principalmente no atendimento de pacientes que vivem em regiões afastadas dos serviços de saúde, sendo, também, uma forma de profissionais especializados apoiarem serviços remotos. (EVISIT, 2020).

A telefarmácia tem demonstrado resultados clínicos positivos nos estudos publicados sobre o tema. Assim como acontece com estudos sobre serviços farmacêuticos presenciais, o impacto desses serviços por vezes é difícil de ser demonstrado, tendo em vista os amplos aspectos que influenciam no cuidado, as distintas ferramentas empregadas na telefarmácia e a dificuldade de se replicar as intervenções individuais e a forma de isolar os resultados obtidos apenas como efeitos exclusivos desse serviço. Conforme revisão sistemática da literatura realizada por Niznik (2008), foram avaliados 34 estudos que mediram os resultados clínicos de intervenções utilizando a telefarmácia. A maioria dos modelos de atendimento ocorreu por agendamento $(n=29)$, sendo o telefone o método de comunicação mais comum $(n=25)$. As intervenções mais utilizadas foram orientações relacionadas com medicamentos $(n=10)$, com 
foco no manejo de doenças crônicas como hipertensão, diabetes, asma, depressão e cessação tabágica em adultos. Vinte e três estudos tiveram um impacto positivo nos desfechos clínicos, com um relatando resultados negativos. Como resultado, foi identificado que as intervenções clínicas de telessaúde em farmácia ambulatorial, principalmente por telefone, têm um impacto geral positivo nos resultados relacionados ao tratamento da doença, autogestão do paciente e adesão ao tratamento de doenças crônicas. As similaridades entre os estudos com impacto positivo incluíram a utilização de modelos programados via telefone, com monitoramento e intervenções frequentes. Os estudos identificados não avaliaram os benefícios da capacidade de vídeo por telefone ou uma avaliação de custo-benefício.

A diferença entre o serviço farmacêutico usual e a telefarmácia é principalmente o espaço em que ocorrem: virtual ou presencial. A forma virtual pode ampliar o atendimento, mas tem atrelada limitações como a diminuição do vínculo e aspectos da comunicação que não são transmitidos a distância. Os demais aspectos relacionados à implementação de um serviço farmacêutico devem ser mantidos e respeitados para que a pessoa receba um serviço de qualidade. A telefarmácia utiliza como base o modelo de cuidado farmacêutico que deve ser centrado na pessoa e suas necessidades. Além disso, é um serviço que deve ser individualizado e respeitar a privacidade de quem o recebe de forma remota.

\section{ESTRATÉGIAS EMERGENTES}

A escola de estratégia mais conhecida no senso comum e predominante até o final dos anos 1970 era a escola de planejamento, fundamentada em uma perspectiva que compreende a estratégia como uma série de atividades deliberadas e materializadas em um plano. $\mathrm{O}$ planejador, neste caso, é um ser capaz de tomar decisões baseadas na leitura de cenários, formalizando-as e executando-as. A responsabilidade pelo processo encontra-se toda com os que assumem o papel de planejar de maneira consciente e controlada (MINTZBERG, 2010).

No final dos anos 1970, Mintzberg (1978) cunhou o termo estratégia emergente, descolando-a do planejamento. A estratégia, para ele, é uma linha padrão de ação coerente ao longo do tempo, conduzindo a organização a objetivos desejáveis, ainda que sem ser guiada por um instrumento formal (MINTZBERG, 1978). A formação da estratégia é o processo de seu desenvolvimento, mais ou menos consciente, ao longo de um curso no qual é moldada pelos envolvidos na organização. São premissas da formação de estratégia: (1) as estratégias são planos para o futuro, mas também são moldadas pelo passado; (2) estratégias não precisam 
ser deliberadas, também podem emergir; (3) estratégias efetivas desenvolvem-se em todos os tipos de caminhos estranhos; (4) reorientações estratégicas acontecem em espaços breves e súbitos; e (5) administrar estratégia é criar pensamento e ação, controle e aprendizado, estabilidade e mudança (MINTZBERG, et. al., 2007).

Avançando no modo como as estratégias emergentes são indissociadas da prática, Mariotto (2003) indica que é preciso reconhecer que elas são fruto de um processo de aprendizagem. Em um nível mais alto, objetivos gerais são (re)definidos e alimentam ações específicas que são executadas e avaliadas, sendo um processo retroalimentado pelo outro. Desta relação emerge a estratégia, sendo papel dos dirigentes estarem abertos a novas possibilidades em seus modelos de negócio, como o caso a seguir demonstra.

\section{O TELECUIDADO FARMACÊUTICO NO ESTADO DO RIO GRANDE DO SUL}

Cuidado farmacêutico é o modelo de prática que orienta a provisão de diferentes serviços farmacêuticos diretamente destinados ao paciente, à família e à comunidade, visando à prevenção e resolução de problemas da farmacoterapia, ao uso racional e ótimo dos medicamentos, à promoção, à proteção e à recuperação da saúde, bem como à prevenção de doenças e de outros problemas de saúde (CONSELHO FEDERAL DE FARMÁCIA, 2016). Nesse sentido, o telecuidado farmacêutico pode ser definido como a prestação de serviços farmacêuticos clínicos de forma remota a uma pessoa, por meio do uso de telecomunicações e outras tecnologias. Considerando esse novo paradigma de oferta de serviços farmacêuticos, bem como a situação atual de pandemia causada pelo novo coronavírus, no dia 5 de maio de 2020 foi lançado pelo do estado do Rio Grande do Sul o Programa de implementação do Cuidado Farmacêutico, CUIDAR+, e o seu primeiro serviço: Telecuidado Farmacêutico. Havia um horizonte de implementar um serviço similar a este, porém a aceleração de sua adoção deveu-se a essa mudança de contexto, emergindo como uma necessidade urgente de resposta à sociedade.

O serviço de orientação sobre o uso correto dos medicamentos, por meio de forma remota, visa acompanhar os pacientes que são atendidos pelas farmácias do Estado. No primeiro momento, o serviço foi lançado para idosos com doenças respiratórias crônicas, pois essa população possuía duplo fator de risco de agravamento à eventual infecção pelo novo coronavírus e cujo agravamento da condição de saúde poderia impactar no aumento das taxas de internações, cenário que competiria com a necessidade de leitos devido à COVID-19. Esta 
primeira etapa foi utilizada como um projeto piloto, oportunizando melhorias nos processos e nos instrumentos de gestão. Como estratégia emergente, o aprendizado se deu na prática, no curso das ações.

O acompanhamento remoto torna-se ainda mais oportuno em um cenário de uma pandemia mundial, cujo devido seguimento se faz ainda mais necessário. Dessa forma, o Telecuidado Farmacêutico complementa as informações já repassadas nas Farmácias e, de uma forma ativa, realiza o acompanhamento da adesão ao tratamento e controle da doença. No Telecuidado Farmacêutico, um profissional farmacêutico capacitado entra em contato com os pacientes com doença respiratória crônica, que receberam medicamentos na Farmácia do Estado, e fornece uma consulta para verificar como está a adesão ao tratamento e o controle da doença. A depender da situação encontrada, a frequência do contato remoto será maior. As intervenções são pactuadas com o paciente e focadas na resolução dos problemas relacionados à farmacoterapia, visando o aumento da efetividade do tratamento e otimização de desfechos clínicos, podendo haver encaminhamentos necessários. Destaca-se, contudo, que o processo de atendimento em si não se visa uma padronização deliberada, dependendo de uma relação específica estabelecida entre o profissional farmacêutico e o usuário do serviço, caracterizando o fluxo de abordagem mais flexível da estratégia emergente.

A telefarmácia tem o potencial de melhorar a qualidade dos serviços farmacêuticos e diminuir os problemas relacionados aos medicamentos. As vantagens adicionais incluem a relação custo-benefício, o acesso ao serviço de orientação sem necessidade de deslocamento do paciente e a possibilidade de atingir pessoas residentes em locais remotos e em condições de saúde que limitam a locomoção. Os serviços de telefarmácia podem ser fornecidos por farmácias comunitárias ou hospitais, por casas de repouso e ambulatórios de atendimento médico. É importante ressaltar que esse serviço deve estar vinculado a um serviço presencial, ser executado por profissional farmacêutico e deve ter foco no usuário.

Alguns desafios são encontrados quando se inicia a oferta desse serviço como, por exemplo, cadastros de usuários desatualizados que impossibilitam o contato e indisponibilidade do usuário de atender o telefone em determinado momento, por não estar familiarizado com a tecnologia ou por condições físicas/cognitivas. A avaliação da implementação do Telecuidado Farmacêutico será realizada por meio de pesquisa em que serão analisados os resultados clínicos dos pacientes atendidos, bem como a satisfação e a viabilidade econômica do serviço. O sistema de registro para evolução das consultas é fundamental para a análise dos dados e 
documentação dos atendimentos. Assim, no Estado, foi adaptado o sistema de dispensação de medicamentos, incluindo o registro das consultas realizadas pelos profissionais farmacêuticos. Além de ações relacionadas ao Telecuidado Farmacêutico, também estão sendo desenvolvidas ações de tele-educação e telemonitoramento para profissionais. É um processo em construção constante, através de uma formação estratégica que está sendo moldada por meio do aprendizado dos envolvidos com os resultados do serviço e de sua execução.

No Brasil, a telefarmácia e os serviços de teleconsulta pelo farmacêutico ainda não estão regulamentados pelo Conselho Federal de Farmácia (CFF), mas percebe-se que essa pauta está cada vez mais presente nas discussões do exercício da profissão. Nesse sentido, o Telecuidado Farmacêutico, uma iniciativa implementada para contribuir no combate à COVID-19, auxiliará na qualificação da regulamentação da telefarmácia no País, a partir da avaliação de sua viabilidade, custos e impacto, visto sua magnitude e por se tratar de uma iniciativa inovadora realizada pela Administração Pública.

Para a teoria, fica mais um registro da importância de reconhecer a estratégia emergente como um desenho estratégico adequado a contextos instáveis e a implementação de serviços no âmbito da gestão em saúde. A concepção fundamentada na lógica do plano deliberado como uma antecipação da execução tem cada vez menos sentido em um mundo dinâmico, no qual a sociedade demanda novas soluções para novos problemas. A moldagem da estratégia emergente tem a capacidade de responder a transformações de nosso tempo de maneira mais efetiva, trazendo benefícios imediatos para os usuários do sistema de saúde.

\section{REFERÊNCIAS}

BRASIL. Lei $\mathrm{n}^{\circ}$ 13.989, de 15 de abril de 2020. Dispõe sobre o uso da telemedicina durante a crise causada pelo coronavírus (SARS-CoV-2). Diário Oficial da União, 2020.

BRASIL. Ministério da Saúde. Portaria MS/GM n 467, de 20 de março de 2020. Dispõe, em caráter excepcional e temporário, sobre as ações de Telemedicina, com o objetivo de regulamentar e operacionalizar as medidas de enfrentamento da emergência de saúde pública de importância internacional previstas no art. $3^{\circ}$ da Lei $n^{\circ} 13.979$, de 6 de fevereiro de 2020, decorrente da epidemia de COVID-19 [Internet]. Diário Oficial da União, Brasília (DF), 2020 mar. Disponível em: <http://www.in.gov.br/en/web/dou/-/portaria-n-467-de-20-de-marco-de2020-249312996>. Acesso em: 27 de nov. de 2020. 
BLUM, K. Around-the-clock review via telepharmacy. Pharmacy Practice News, 2016. Disponível em: <http://www.pharmacypracticenews.com/Technology/Article/10-16/Aroundthe-ClockReview-Via-Telepharmacy/38231/ses1/4ogst>. Acesso em: 27 de nov. de 2020.

CONSELHO FEDERAL DE FARMÁCIA (CFF). Serviços farmacêuticos diretamente destinados ao paciente, à família e à comunidade: contextualização e arcabouço conceitual. 2016. Disponível em: <http://www.cff.org.br/userfiles/Profar_Arcabouco_TELA _FINAL.pdf>. Acesso em: 27 de nov. de 2020.

EVISIT. Disponível em: 〈https://evisit.com/resources/what-is-telepharmacy/>. Acesso em 12 Nov. 2020.

LE, Tin; TOSCANI, Michael; COLAIZZI, John. Telepharmacy: A New Paradigm for Our Profession. Journal of Pharmacy Practice, v. 33, n. 2, p. 176-182, 2020.

MARIOTTO, F. L. Estratégia: Mobilizando Estratégias Emergentes. Revista RAE, v. 43, 2003.

MINTZBERG, H. Patterns in strategy formation. Management Science, v. 29, n. 9, p. 934-48, 1978

MINTZBERG, Henry; AHLSTRAND, Bruce; LAMPEL, Joseph. Safári da estratégia. Bookman Editora, 2009.

MINTZBERG, H. et al.. O processo da Estratégia: conceitos, contextos e casos selecionados. Porto Alegre: Artmed, 2007.

NIZNIK, Joshua D.; HE, Harvey; KANE-GILL, Sandra L. Impact of clinical pharmacist services delivered via telemedicine in the outpatient or ambulatory care setting: A systematic review. Research in Social and Administrative Pharmacy, v. 14, n. 8, p. 707-717, 2018.

RS lança serviço de Telecuidado Farmacêutico voltado a idosos com doenças respiratórias. Portal Saúde RS. Porto Alegre, 5, maio, 2020. Disponível em: <https://saude.rs.gov.br/estadolanca-servico-de-telecuidado-farmaceutico-voltado-a-idosos-com-doencas-respiratorias>. Acesso em 27 de nov. de 2020.

TRAYNOR, Kate. Navy takes telepharmacy worldwide. 2010. 
WORLD HEALTH ORGANIZATION. Resolution. Fifty-eighth World Health Assembly, Geneva, p. 16-25, 2005. Disponível em: <http://apps.who.int/gb/ ebwha/pdf_files/WHA58REC1/english/A58_2005_REC1-en. pdf>. Acesso em 28 nov. 2020. 